Bearder, S.K. 279, 300

Bettridge, C.M. 319

Butynski, T.M. 300, 319

de Jong, Y.A. 300, 319

de Kort, S.R. 319

delBarco-Trillo, J. 422

Disotell, T.R. 279

Drea, C.M. 422

Gamba, M. 279

Geerah, D.R. 392

Goodwin, T.E. 422
Grow, N.B. 404

Gursky, S. 273, 379

Hending, D. 361

Holderied, M. 336

Karlsson, J. 279

Kenworthy, S.P. 319

Mandl, I. 336

Nekaris, K.A.I. 273, 392

Nijman, V. 300
O’Hagan, R.P. 392

Perkin, A. 279

Pozzi, L. 279

Schneiderová, I. 300

Schwitzer, C. 336

Seiler, M. 361

Stanger-Hall, K.F. 361

Svensson, M.S. 300

Wirdateti, W. 392

\title{
Subject Index Vol. 90, No. 5, 2019
}

Active acoustics 392

Advertisement call(s) 300, 319

Africa 279

Alarm call 319

Antipredator strategy 404

Behavioural ecology 422

Bioacoustics 300, 319

Biogeography 300

Body size 404

Bushbaby 300, 319

Call gradation 336

Cathemerality 422

Chemical analysis 422

Communication 273, 319, 404

Crypsis 404

Cryptic species 279

Cytochrome b 279

Diurnality 422
Evolution 422

Gas chromatography-mass spectrometry 422

Indonesia 379

Isolation by distance 300

Lepilemur 336

Lemur 422

Lorisiformes 392

Mate attraction 361

Mirza zaza 361

Mitochondrial DNA 279

Nocturnal primates 319, 336

Nocturnality 422

Northern giant mouse lemur 361

Oestrus cycle 361

Olfaction 273

Olfactory communication 422
Population differences 319

Primate(s) 273, 379

- communication 392

Prosimian 273

Social interactions 336

Spacing 361

Speciation 279

Strepsirrhini 273, 336

Tarsius 379, 404

Ultrasonic calls 379

- vocalisation 273, 404

Ultrasound 392

Urine marking 422

Vocal communication 336

Vocalisation(s) 279, 300, $319,361,379$ 